\title{
Study of Fluctuation Suppression during Potential Formation in the Tandem Mirror GAMMA 10
}

\author{
Masayuki YOSHIKAWA, Yoshiaki MIYATA, Masanori MIZUGUCHI, Neo IMAI, \\ Hitoshi HOJO, Makoto ICHIMURA, Tsuyoshi KARIYA, \\ Isao KATANUMA, Yousuke NAKASHIMA, Ryutaro MINAMI, Hiroyuki SHIDARA, \\ Yuusuke YAMAGUCHI, Yoriko SHIMA, Youhei OONO, Fumiaki YAGUCHI \\ and Tsuyoshi IMAI \\ Plasma Research Center, University of Tsukuba, Tsukuba, Ibaraki 305-8577, Japan
}

(Received 5 December 2009 / Accepted 13 May 2010)

\begin{abstract}
In the tandem mirror GAMMA 10, plasma confinement is achieved by not only a magnetic mirror configuration but also high potentials in both end regions. Before plug electron cyclotron resonance heating (P-ECH) was applied, drift-type fluctuations were observed in potential and density measurements. Potential and density fluctuation suppression during potential formation by the application of P-ECH was clearly observed using a gold neutral beam probe (GNBP) system. We study the relationship between the suppression levels of potential fluctuations and the effects of the produced potentials by varying the applied ECH power. Moreover, the particleflux-related values obtained by the phase difference between the potential and density fluctuations are measured using the GNBP. We can clearly show that radial anomalous transport induces radial particle transport, which decreases the plasma stored energy.
\end{abstract}

(C) 2010 The Japan Society of Plasma Science and Nuclear Fusion Research

Keywords: Tandem mirror GAMMA 10, gold neutral beam probe, potential, electric field, fluctuation

DOI: $10.1585 /$ pfr.5.S2010

\section{Introduction}

In the tandem mirror GAMMA 10, an electrostatic potential for improving axial confinement is created by applying electron cyclotron resonance heating $(\mathrm{ECH})$ in the end mirrors (plug/barrier cells) [1-6]. Two types of instability, a rotationally driven mode with the lowest azimuthal mode number and a drift-wave mode with high azimuthal mode numbers, are observed during the existence of confining potentials. The rotational mode is driven by $E \times B$ rotation energy at the lowest azimuthal mode number, $m$ $=1$, in tandem mirrors. Higher modes with $m>2$ will be stabilized by finite Larmor radius effects. On the other hand, the drift-wave mode arises because of the existence of a density gradient. The radial electric field $E$ arising from the potential causes an $E \times B$ plasma rotation in the direction of the ion diamagnetic drift velocity, which may enhance instabilities, such as rotational flute and drift-wave modes, and degrade radial confinement. However, present studies show the suppression of low-frequency fluctuations of the density and the potential during axial confining potential formation during plug-ECH (P-ECH) [1-6]. Earlier works by Sanuki [7] and Chaudhry et al. [8] discussed the effects of the ambipolar potential on the stability of drift waves. The stability is considered to be due to ion Landau damping caused by the velocity shear effect of the
$E \times B$ drift. The density fluctuation is observed using microwaves, for example, by interferometry, reflectometry, and the Fraunhofer diffraction method, and electrostatic probes $[1-3,5,6]$. GAMMA 10 has a good potential measurement system consisting of a gold neutral beam probe (GNBP) system [4, 6,9]. We can observe density and potential fluctuations during axial confinement potential formation when P-ECH is applied in a single plasma shot. In previous experiments, we could measure only the potential and its fluctuation at one plasma radial position. We tried to obtain the potential and its fluctuation at two radial positions in a single plasma shot by applying a rectangular wave form at the vertical beam deflector electrode. After successfully obtaining these, we then obtained the radial electric field profile. We observed the suppression of density and potential fluctuations by potential formation during P-ECH by using the GNBP. This allows us to study the suppression mechanisms of drift-type fluctuations during the formation of a confinement potential produced by $\mathrm{ECH}$ in greater detail.

In this paper, we show the phase differences between the electron density fluctuation and the potential fluctuation in order to study the radial particle-flux-related value. Moreover, we show the relationships between the suppression of drift-type potential fluctuations and the radial profiles of the potential and electric field. 


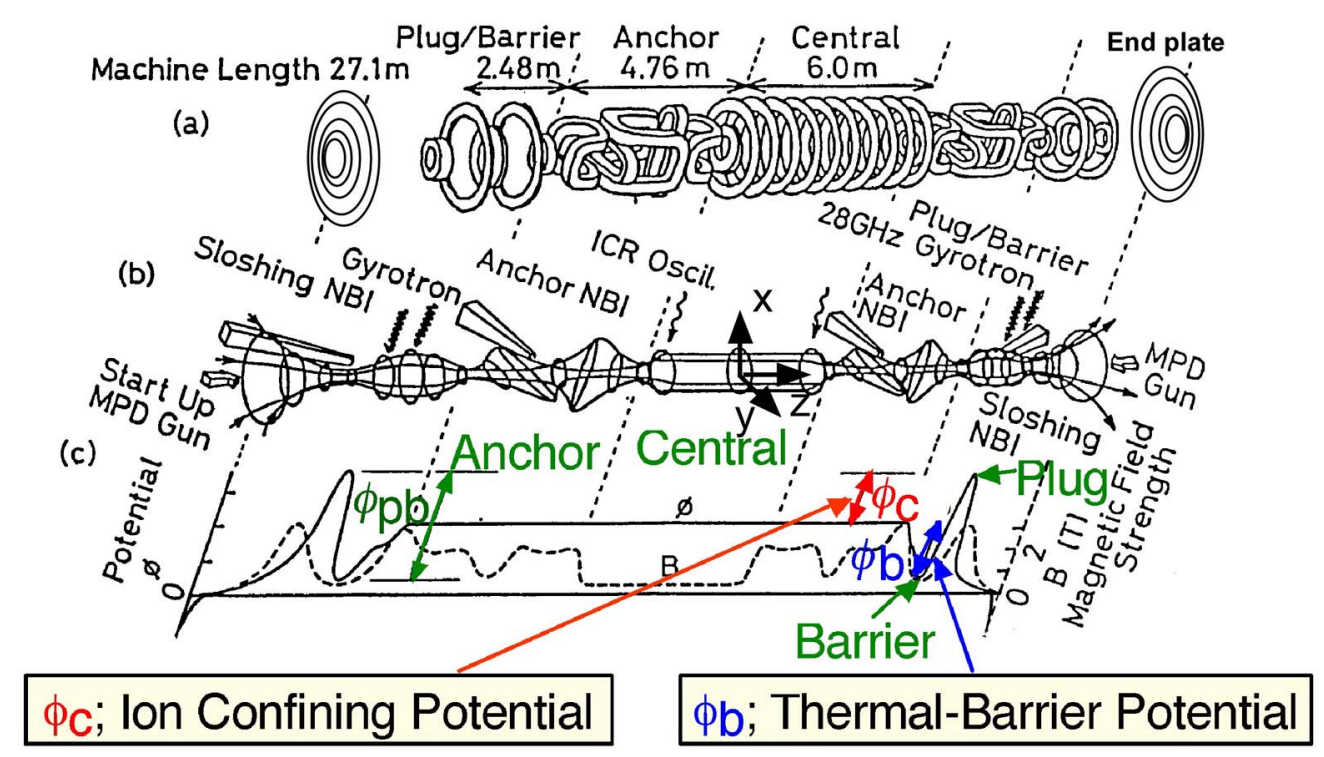

Fig. 1 Schematic of tandem mirror GAMMA 10.

\section{Experimental Apparatus}

\subsection{Tandem mirror GAMMA 10}

GAMMA 10 is an effectively axisymmetrized minimum-B anchored tandem mirror with thermal barriers at both end mirrors (Fig. 1). The details on GAMMA 10 are provided elsewhere [1-6]. The $x$-axis and $y$-axis are perpendicular to the magnetic field in the vertical and horizontal directions, respectively. The $z$-axis is parallel to the magnetic field. In the tandem mirror GAMMA 10, the central, anchor, and plug/barrier cells are 6.0-, 4.8-, and $2.5-\mathrm{m}$ long, respectively. The magnetic field strength at the mid-plane of the central cell is $0.41 \mathrm{~T}$ during standard operation, and the mirror ratio is 5 . The anchor cells are located on both sides of the central cell and consist of a minimum-B mirror field that is produced by a baseball coil. The magnetic field strength is $0.61 \mathrm{~T}$ at the mid-plane of the anchor cell, and the mirror ratio is 3. Plug/barrier cells are located at both ends of GAMMA 10, where the electron confinement and ion confinement potentials are produced. The difference between the central potential $\left(\Phi_{\mathrm{c}}\right)$ and the plug potential $\left(\Phi_{\mathrm{p}}\right)$ is the ion confinement potential $\left(\phi_{\mathrm{p}}\right)$. The difference between the central potential $\left(\Phi_{\mathrm{c}}\right)$ and the barrier potential $\left(\Phi_{\mathrm{b}}\right)$ is the thermal barrier potential $\left(\phi_{\mathrm{b}}\right)$. The plasma is created by plasma guns and heated and sustained using ion cyclotron range of frequency (ICRF) heating systems. Three types of oscillators are used: RF1, RF2, and RF3. Waves excited by the RF1 and RF2 systems cause plasma production and ion heating in the central cell, respectively. As the applied P-ECH power is increased from 150 to $300 \mathrm{~kW}$, the plug potential can be changed from about 1.5 to $1.8 \mathrm{kV}$ in these experiments. The ion and electron axial confinement potentials are produced using P-ECH and barrier-ECH (B-ECH) at both end mirror regions (plug/barrier cells). The typical electron density, electron temperature, and ion temperature are about $2 \times 10^{12} \mathrm{~cm}^{-3}, 0.1 \mathrm{keV}$, and $5 \mathrm{keV}$, respectively.

\subsection{Gold neutral beam probe}

We use the heavy ion beam probe (HIBP) to measure the potential of the core plasma at the central cell in GAMMA 10. We measured the potential and its fluctuations using the GNBP in the central cell (Fig. 2) [9]. In a vertically directed beam sweep for radial potential profile observations in a single discharge, ionization points move in the vertical direction due to varying incident angles. The GNBP uses a neutral primary beam and negative gold ions formed by Cs sputtering [4]. The energy and incident angle of the primary beam passing the plasma center are about $12 \mathrm{keV}$ and 40 degrees, respectively. A typical primary beam current of $2 \mu \mathrm{A}$ is obtained using a Faraday cup. The GNBP system has two incident angle electrostatic deflectors for the vertical and horizontal directions, respectively. A parallel-plate-type electrostatic energy analyzer with an incident angle of 45 degrees is installed in the $x-y$ plane. The analyzer uses a microchannel plate detector with 32 anodes mounted along the $y$ direction as a beam detector. The detected positive secondary beam is derived from the neutral primary beam ionized at the ionization position. The electron-impact ionization process is dominant in ionization of the primary beam. The secondary beam current depends on the electron distribution function at the ionization position. The density fluctuation is obtained from the perturbation of the detected beam intensity, and the potential fluctuation is obtained from the perturbation of the plasma potential. It is possible to simultaneously measure the potential and density fluctuations and their phase difference at an arbitrary point by the GNBP. The GNBP can measure the radial potential profile from $R \sim 0 \mathrm{~cm}$ to $R \sim 14 \mathrm{~cm}$ with an error of $\pm 10 \mathrm{~V}$. The path integral effect is low in the density and potential fluc- 


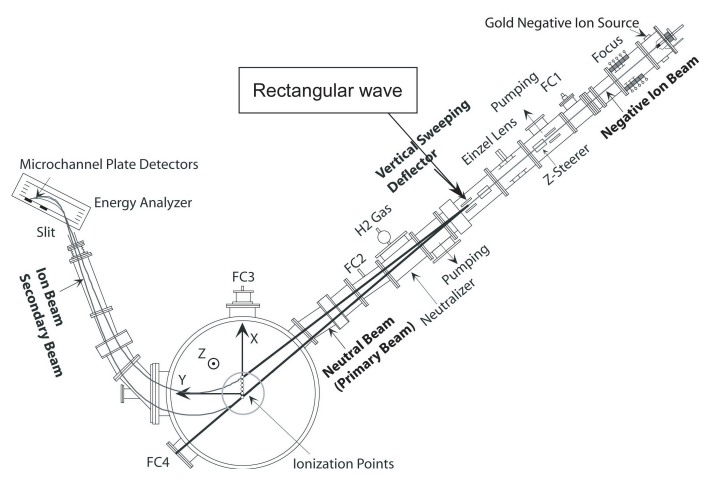

Fig. 2 GNBP system in GAMMA 10.

tuations measured in the density range and low fluctuation levels of the GAMMA 10 experiment $[10,11]$.

\section{Fluctuation Measurements}

\subsection{Particle-flux-related values analyzed by GNBP}

We observed potential and relative density fluctuation frequencies of about $8-12 \mathrm{kHz}$, which correspond to drifttype fluctuations. We concentrated on the period without P-ECH. Figure 3 shows the diamagnetism indicated by a solid blue line and line density indicated by a dotted red line. Figure 4 shows the temporal evolution of the low-frequency potential and density oscillations near the center $(r \sim 0 \mathrm{~cm})$. The red dotted line indicates the density fluctuation, and the blue line indicates the potential fluctuation levels. Anomalous transport is said to originate in particle flux loss induced by drift-wave-type instabilities. The phase difference between the potential and density fluctuations is a crucial in whether these fluctuations induce anomalous particle transport. The particleflux-related value was evaluated from the potential and density fluctuations and their phase difference measured by the GNBP. In GAMMA 10, azimuthally propagating electrostatic fluctuations are observed. The radial particleflux-related value for experimental investigation is derived as, $\Gamma_{\mathrm{P}} \approx \frac{2}{B} \int_{0}^{\infty} k_{\theta}\left|\gamma_{n \phi}\right| \tilde{n} \tilde{\phi} \sin \alpha_{n \phi} \mathrm{d} \omega$, where $k_{\theta}, \gamma_{n \phi}, \tilde{n}, \tilde{\phi}, \alpha_{n \phi}$ are wave number, coherence between density and potential fluctuations, density and potential fluctuations, and phase difference between the density and potential fluctuations, respectively. The observed potential and density fluctuation frequencies are about $10-12 \mathrm{kHz}$. These fluctuations correspond to diamagnetic drift-type fluctuations. A good correlation between the oscillations is recognized. Figure 5 shows the time evolution of the diamagnetism (dotted line) and radial flux estimated from these fluctuations at $R \sim 2 \mathrm{~cm}$ (solid line). The diamagnetism began to decrease at $120 \mathrm{~ms}$, when the particle flux was in an increasing phase. When the particle-flux-related value was in a decreasing phase at $125 \mathrm{~ms}$, the diamagnetism entered an increasing phase. The estimated particle-flux-related value

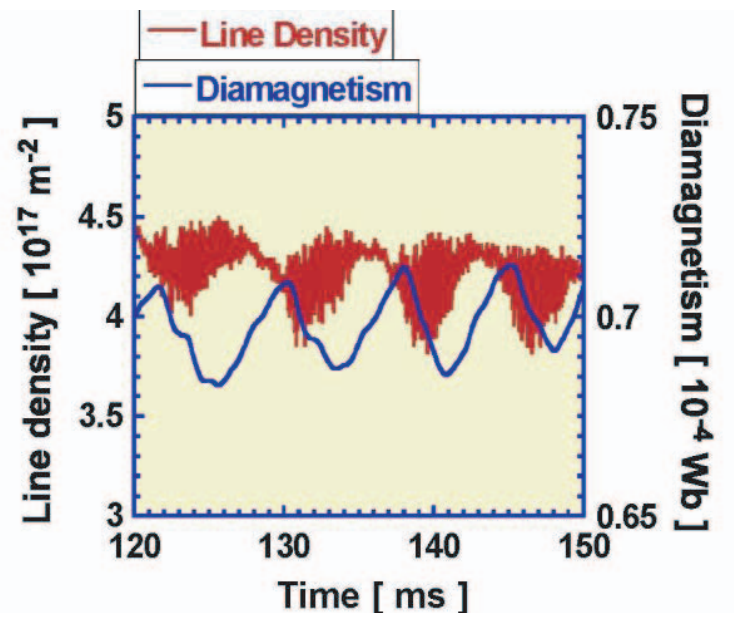

Fig. 3 Temporal evolution of line density (red line) and diamagnetism (blue line).

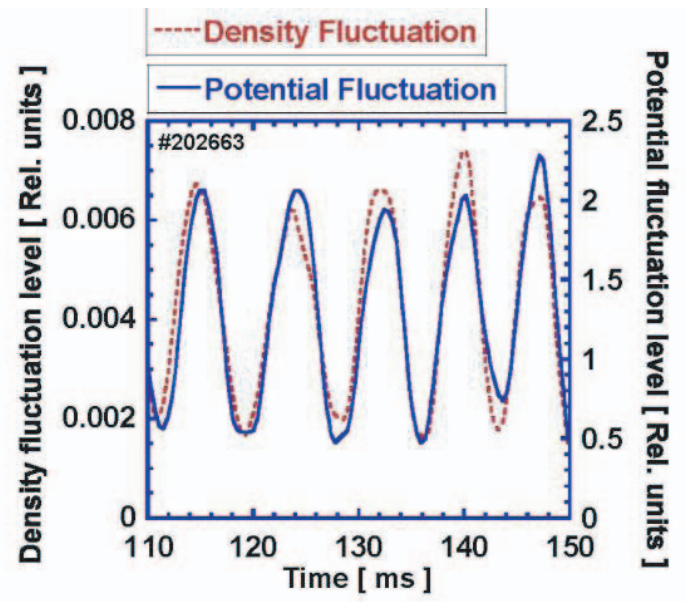

Fig. 4 Temporal evolution of density fluctuation (red dotted line) and potential fluctuation (blue solid line).

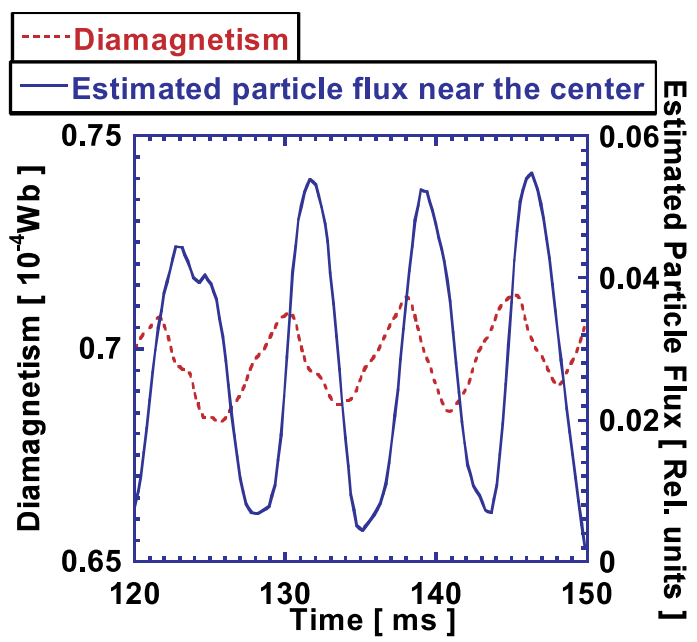

Fig. 5 Time evolution of diamagnetism (red dotted line) and estimated particle-flux-related value near the center (blue solid line). 

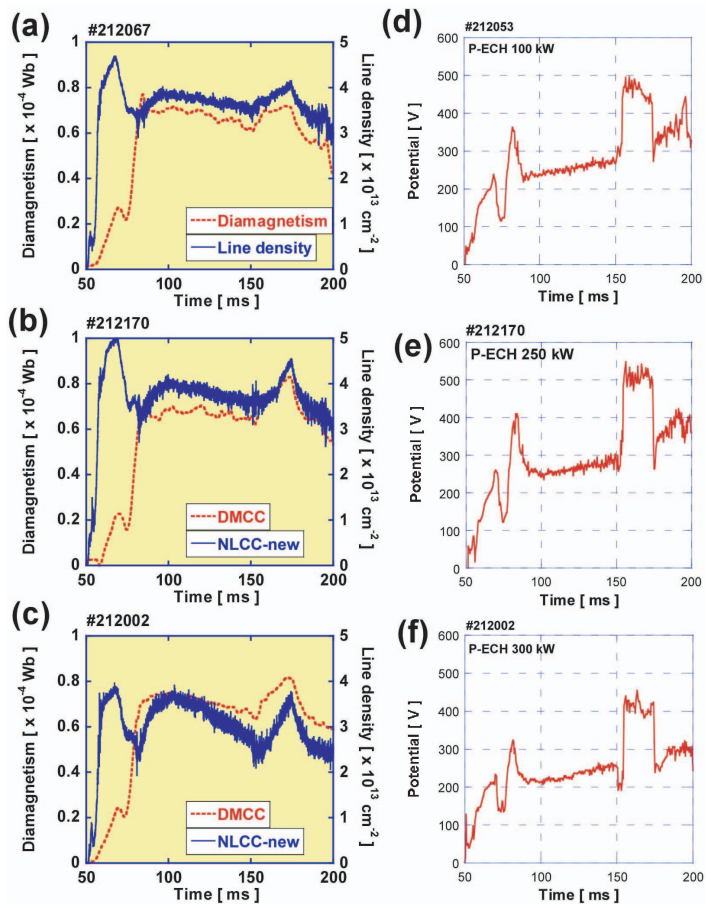

Fig. 6 Temporal evolution of line density (blue solid line) and diamagnetism (red dotted line) in (a), (b), and (c), and potentials in (d), (e), and (f) with P-ECH powers of 100 , 250 , and $300 \mathrm{~kW}$, respectively.

of about 0.035 is the threshold of the increasing and decreasing phases of the diamagnetism.

\subsection{Potential fluctuation suppression during potential formation}

We used the GNBP system to measure the radial potential and electric field profiles. We applied a rectangular wave to the deflector electrodes to measure the plasma potentials at two positions in a single plasma shot. We varied the P-ECH power to obtain different axial confining potential conditions. With a large P-ECH power, we obtained a larger plug potential. Figure 6 shows the temporal evolution of line density (blue solid line) and diamagnetism (red dotted line) [(a), (b), and (c)] and the potentials [(d), (e), and (f)] with P-ECH powers of 100, 250, and $300 \mathrm{~kW}$, respectively. B-ECH and $\mathrm{P}-\mathrm{ECH}$ were applied from 155 to $200 \mathrm{~ms}$ and 155 to $175 \mathrm{~ms}$, respectively, to produce an axial confining potential. The central potential quickly increased in the P-ECH period. When we applied the vertical rectangular deflector voltage on the GNBP, the central cell potential was measured at $R \sim 11$ and $13 \mathrm{~cm}$ in a single plasma shot [Fig. 7 (a)]. Figure 7 (b) shows the central cell potentials obtained at two radial positions. We varied the applied vertical rectangular deflector voltage to measure the region from $R \sim 1$ to $14 \mathrm{~cm}$ so as to obtain radial profiles of the potential and electric field. Figure 8 shows the radial potential profiles [(a), (b), and (c)] and the radial electric field profiles [(d), (e), and (f)] at P-ECH powers of 100,250 , and $300 \mathrm{~kW}$, respectively. The red closed circles
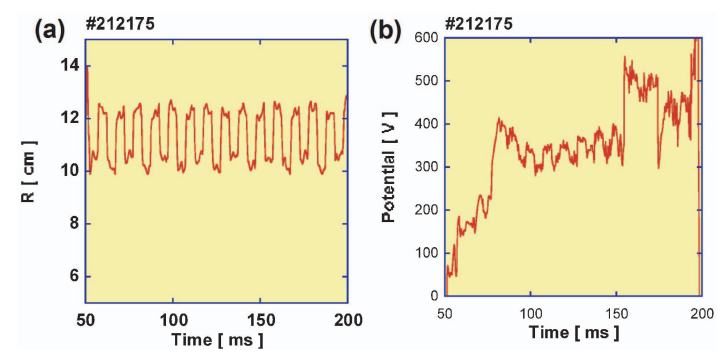

Fig. 7 Applied rectangular vertical deflector voltage (a) and observed potential at two plasma positions (b).
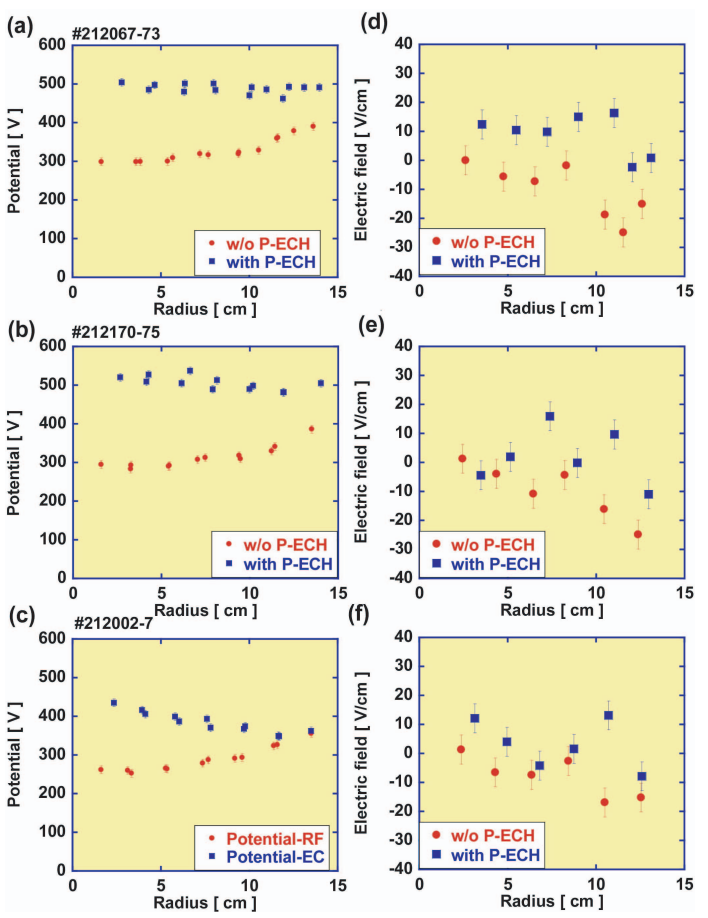

Fig. 8 Radial potential profiles at P-ECH powers of $100 \mathrm{~kW}$ (a), $250 \mathrm{~kW}(\mathrm{~b})$, and $300 \mathrm{~kW}$ (c), and radial electric field profiles at P-ECH powers of $100 \mathrm{~kW}(\mathrm{~d}), 250 \mathrm{~kW}$ (e), and $300 \mathrm{~kW}$ (f).

and blue closed squares indicate the radial potential profile without and with P-ECH, respectively. We obtained the electric fields by using the differences between the average potential values at $t=140.833$ and $149.916 \mathrm{~ms}$ and the potential value at $t=145.375 \mathrm{~ms}$ before P-ECH application, and the differences between the average potential values at $t=160.513$ and $169.597 \mathrm{~ms}$ and the potential value at $t=165.055 \mathrm{~ms}$ during P-ECH application. As the applied P-ECH power increased from 150 to $300 \mathrm{~kW}$, the plug potential changed from about 1.5 to $1.8 \mathrm{kV}$ in these experiments. However, the central potential did not linearly depend on the P-ECH power. Thus, the radial potential and electric field profiles did not linearly depend on the P-ECH power. Before the application of P-ECH, the potential at the plasma center $(R \sim 2 \mathrm{~cm})$ was about 290 , 295 , and $260 \mathrm{~V}$ at P-ECH powers of 100,250 , and $300 \mathrm{~kW}$, respectively. During the application of $\mathrm{P}-\mathrm{ECH}$, the poten- 

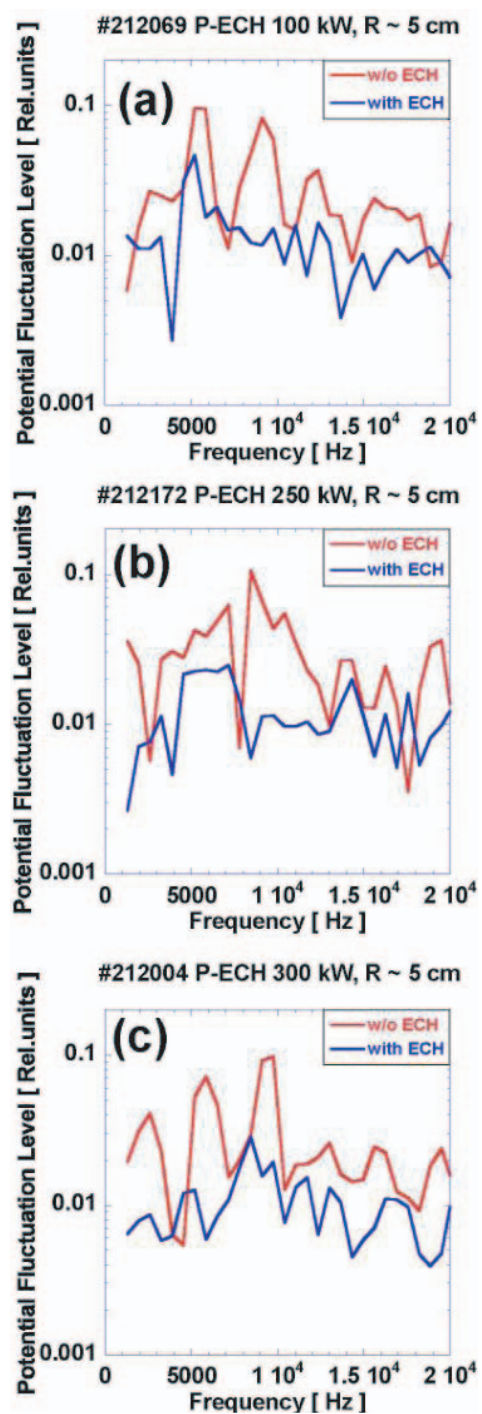

Fig. 9 Frequency spectra of potential fluctuation at $R \sim 5 \mathrm{~cm}$ with P-ECH powers of $100 \mathrm{~kW}$ (a), $250 \mathrm{~kW}$ (b), and $300 \mathrm{~kW}(\mathrm{c})$.

tial at the plasma center $(R \sim 2.5 \mathrm{~cm})$ was about 500, 520, and $435 \mathrm{~V}$ at P-ECH powers of 100,250 , and $300 \mathrm{~kW}$, respectively. Before P-ECH was applied, the radial potential profile showed a lower potential at the plasma center $(R \sim 0 \mathrm{~cm})$ than at $R \sim 14 \mathrm{~cm}$. With the application of $\mathrm{P}-\mathrm{ECH}$, the radial potential profile showed a higher potential at the plasma center $(R \sim 0 \mathrm{~cm})$ than at $R \sim 14 \mathrm{~cm}$. The electric field strengths at $R>11 \mathrm{~cm}$ are not the same as those at $R<11 \mathrm{~cm}$. Before application of P-ECH, a negative electric field was observed, whereas during $\mathrm{P}$ ECH application, a positive electric field was obtained at $R<11 \mathrm{~cm}$. However, a negative electric field greater than $-5 \mathrm{~V} / \mathrm{cm}$ was observed at some positions. Potential fluctuation spectra at P-ECH powers of 100, 250, and $300 \mathrm{~kW}$ are shown in Figs. 9 (a), (b), and (c), respectively. The red and blue lines indicate the frequency spectrum without and with P-ECH at $R \sim 5 \mathrm{~cm}$, respectively. Potential fluctuation suppression of spectra around frequencies of $8-12 \mathrm{kHz}$ was clearly observed with the application of P-ECH. A fre-
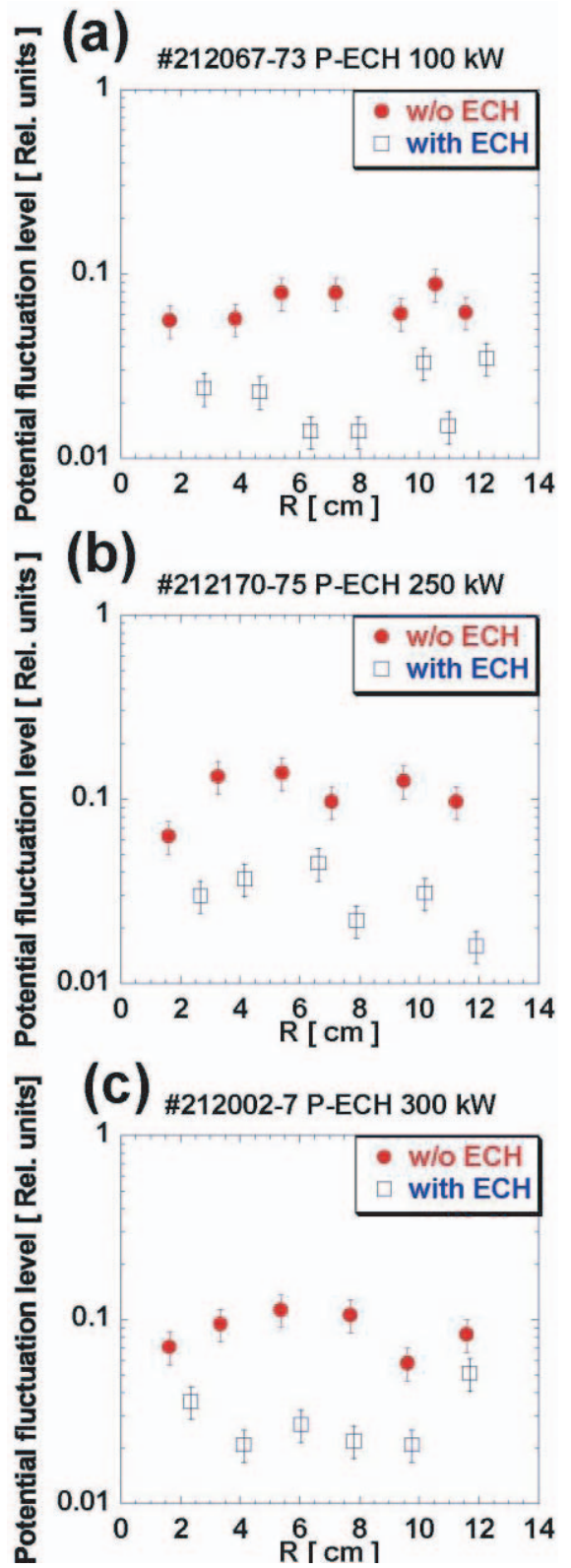

Fig. 10 Potential fluctuation peak in radial profiles around frequencies of $8-12 \mathrm{kHz}$ with P-ECH powers of $100 \mathrm{~kW}$ (a), $250 \mathrm{~kW}(\mathrm{~b})$, and $300 \mathrm{~kW}(\mathrm{c})$.

quency of $10 \mathrm{kHz}$ corresponds to diamagnetic drift-type fluctuation. Figures $10(\mathrm{a}),(\mathrm{b})$, and (c) show the potential fluctuation peak in radial profiles around frequencies of $8-12 \mathrm{kHz}$ with $\mathrm{P}-\mathrm{ECH}$ powers of 100,250 , and $300 \mathrm{~kW}$, respectively. Closed red circles and open blue squares indicate the frequency spectra without and with $\mathrm{P}-\mathrm{ECH}$, respectively. The smallest level of the measurable potential fluctuation is about 0.012. Potential fluctuation suppression with the application of P-ECH was clearly observed in the core region $(R<10 \mathrm{~cm})$.

\section{Discussion}

We analyzed the particle-flux-related value using the GNBP. The estimated particle-flux-related value and the 
diamagnetism are correlated well at the center of the central cell. The edge region must be considered in a detailed particle flux analysis. The frequency of the diamagnetism and estimated particle-flux-related value oscillation is about $100 \mathrm{~Hz}$. The fluctuation frequency of very low frequency oscillation (about $100 \mathrm{~Hz}$ ) is about $10 \mathrm{kHz}$. It was the oscillation of the drift type fluctuation oscillation. We confirmed this by examining the phase difference between the two electrostatic probes installed at the two azimuthal positions. The reason for the low-frequency oscillation was not clear, but we believe that the $100-\mathrm{Hz}$ oscillation may depend on the relaxation time of the fluctuations. The end-loss ion current measured by the end-loss energycomponent analyzer [12] installed in the end region was constant. This indicates that one source of the anomalous radial transport in the central region is the potential and density fluctuations and that the fluctuations decrease the plasma stored energy.

Moreover, we measured the radial potential and electric field profiles by using the GNBP by applying a rectangular wave to the vertical deflector electrodes for the first time. Limiters with a central cell conversion radius of $11.5 \mathrm{~cm}$ are installed at $z= \pm 733.5 \mathrm{~cm}$ to improve the efficiency of the P/B-ECH waves in ICRF plasma heating. For these experiments, we concentrate our discussion on the region of $R<11.5 \mathrm{~cm}$. The electric field strengths at $R>11 \mathrm{~cm}$ are not the same as those at $R<11 \mathrm{~cm}$. Before application of P-ECH, negative electric fields were observed, whereas during P-ECH application, positive electric fields were obtained at $R<11 \mathrm{~cm}$. However, a negative electric field larger than $-5 \mathrm{~V} / \mathrm{cm}$ was observed at a few positions. However, since the electric field measurements have an error of about $\pm 5 \mathrm{~V} / \mathrm{cm}$, the negative electric fields observed during P-ECH application may not actually be negative. Measurements of the potential fluctuations reveal that the fluctuation power of coherent modes $(8-12 \mathrm{kHz})$ is suppressed in the axially confined potential with P-ECH application. A frequency peak of the coherent mode of about $9 \mathrm{kHz}$, corresponding to diamagnetic drift, is observed before P-ECH application. When P-ECH was applied, non-drift-type fluctuation increased. Drifttype fluctuation was caused by drift waves and is a global mode fluctuation. The observed drift-type fluctuation is the diamagnetic drift during the period without P-ECH; it occurred at positions with large density gradient. Drifttype fluctuation drives the plasma rotation at the same fluctuation frequency at all radial positions in GAMMA 10. Drift-type fluctuation near the center was also observed in GAMMA 10. Figure 11 shows the relationship between the potential and the potential fluctuation levels. The potential fluctuation levels decrease with increasing potential. Figure 12 shows the relationship between the electric field strength and the potential fluctuation. The closed circles, squares, and triangles represent the fluctuation levels without P-ECH powers of 100,250 , and $300 \mathrm{~kW}$, respectively. The open circles, squares, and triangles rep-

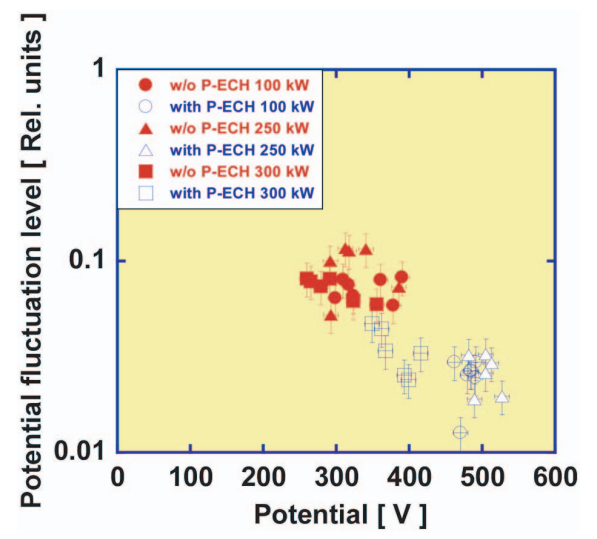

Fig. 11 Relationship between potential fluctuation and potential.

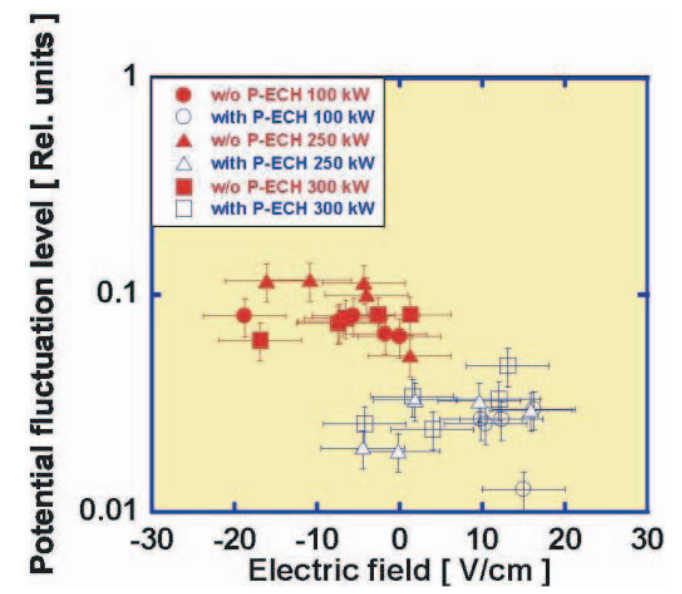

Fig. 12 Relationship between potential fluctuation and electric fields.

resent the fluctuation levels with P-ECH powers of 100 , 250 , and $300 \mathrm{~kW}$, respectively. This indicates that the fluctuation powers are larger in the negative electric field region and smaller in the positive electric field region. Thus, drift-type fluctuations are suppressed by potential formation and positive electric field formation in the central cell. Considering the drift-type fluctuation growth rate, increase in electron temperature causes a higher fluctuation growth rate, and increase in ion temperature causes greater stabilization of drift waves. In GAMMA 10, during potential formation due to $\mathrm{P}-\mathrm{ECH}$, the electron temperature increases from 0.05 to about $0.10 \mathrm{keV}$, and the ion temperature is maintained at about $5 \mathrm{keV}$. The stabilization by the ion temperature is small. The electron density profile before and during $\mathrm{P}-\mathrm{ECH}$ periods remains almost the same. During the periods without $\mathrm{P}-\mathrm{ECH}$, the negative electric fields enhance the diamagnetic drift. During the periods with P-ECH, the positive electric fields suppress diamagnetic drift. The relationships between the suppression of potential fluctuation and the potential and the suppression of fluctuation by the positive electric field are clearly observed. The threshold of the drift-type fluctuation sup- 
pression by the electric field is about $2 \mathrm{~V} / \mathrm{cm}$ according to Hatakeyama's study [13-15]; our results are comparable to his. The $E \times B$ drift shear may suppress drift-type fluctuations. However, in this experiment, the measured electric fields have a large error (about $\pm 5 \mathrm{~V} / \mathrm{cm}$ ). Therefore, the details of the relationship between the electric field shear and suppression of drift-type fluctuation could not be obtained.

\section{Summary}

We analyze the phase differences between the radial positions of electron density fluctuation and potential fluctuation to study the radial particle flux. The estimated particle-flux-related value and the diamagnetism are correlated well at the center of the central cell. This correlation indicates that one source of the anomalous radial transport at the center is the potential and density fluctuations and that the fluctuations decrease the plasma stored energy. Moreover, we show the relationships between the suppression of drift-type potential fluctuation and the potential and electric field. Greater potential formation and a positive electric field suppress drift-type fluctuations.

\section{Acknowledgments}

The authors thank the members of the GAMMA 10 group of the University of Tsukuba for their collaboration. This work was supported in part by the Ministry of Education, Culture, Sports, Science and Technology, Grant-in-Aid for Scientific Research in Priority Area No. 16082203, and the NIFS collaboration research program, No. NIFS04KUGM009.

[1] A. Mase, J. H. Jeong, A. Itakura, K. Ishii, M. Inutake and S. Miyoshi, Phys. Rev. Lett. 64, 2281 (1990).

[2] A. Mase, J. H. Jeong, A. Itakura, K. Ishii and S. Miyoshi,
Rev. Sci. Instrum. 61, 1247 (1990).

[3] A. Mase, A. Itakura, M. Inutake, K. Ishii, J. H. Jeong, K. Httori and S. Miyoshi, Nuclear Fusion 31, 1725 (1991).

[4] Y. Miyata, T. Cho, M. Yoshikawa, Y. Nakashima, M. Hirata, N. Kaidou, H. Kakiuchi, Y. Motegi, S. Goshu, M. Mizuguchi, T. Numakura and J. Kohagura, Plasma Fusion Res. 2, S1101 (2007).

[5] M. Yoshikawa, Y. Shima, T. Matsumoto, A. Nakahara, N. Yanagi, A. Itakura, H. Hojo, T. Kobayashi, K. Matama, Y. Tatematsu, T. Imai, J. Kohagura, M. Hirata, Y. Nakashima and T. Cho, Rev. Sci. Instrum. 77, 10E906 (2006).

[6] M. Yoshikawa, T. Matsumoto, Y. Shima, A. Nakahara, N. Yanagi, A. Itakura, H. Hojo, Y. Kubota, T. Kobayashi, Y. Higashizono, Y. Nakashima, Y. Tatematsu, T. Imai and T. Cho, Plasma Fusion Res. 2, S1036 (2007).

[7] H. Sanuki, Phys. Fluids 27, 2500 (1984).

[8] M. B. Chaudhry, H. Hojo, T. Watanabe and K. Nishikawa, J. Phys. Soc. Jpn. 57, 3043 (1988).

[9] Y. Miyata, M. Yoshikawa, M. Mizuguchi, Y. Oono, F. Yaguchi, N. Matsuda, H. Murata, M. Ichimura, T. Murakami and T. Imai, J. Plasma Fusion Res. SERIES 9, 084 (2010).

[10] A. Kojima, K. Ishii, Y. Takemura, K. Hagisawa, Y. Miyata, A. Itakura, M. Ichimura and T. Cho, J. Plasma Fusion Res. SERIES 6, 677 (2004).

[11] A. Kojima, K. Ishii, Y. Miyata, T. Yamaguchi, S. Fujimoto, H. Kakiuchi, N. Kaidou, H. Higaki, M. Yoshikawa, A. Itakura, M. Ichimura and T. Cho, Journal of the Korean Physical Society 49, S100 (2006).

[12] K. Ishii, T. Goto, Y. Goi, N. Kikuno, M. Nagasaki, Y. Ono, N. Ishibashi, M. Yamanashi, M. Nakamura, I. Katanuma, A. Mase, M. Ichimura, A. Itakura, T. Tamano and K. Yatsu, Phys. Rev. Lett. 83, 3438 (1999).

[13] R. Hatakeyama, T. Kaneko and H. Tsunoyama, J. Plasma Fusion Res. 80, 299 (2004), in Japanese.

[14] M. Yoshinuma, M. Inutake, R. Hatekeyama, T. Kaneko, H. Hattori, A. Ando and N. Sato, Phys. Lett. A 254, 301 (1999).

[15] M. Yoshinuma, K. Hattori, A. Ando, M. Inutake, T. Kaneko, R. Hatakeyama and N. Sato, Tran. Fusion Technol. 35, 278 (1999). 